\title{
ON CERTAIN GROUPS OF FUNCTIONS
}

\author{
J.S. YANG \\ Department of Mathematics, \\ Computer Science, and Statistics \\ University of South Carolina \\ Columbia, South Carolina 29208 U.S.A. \\ (Received October 18, 1979)
}

ABSTRACT. Let $\mathrm{C}(\mathrm{X}, \mathrm{G})$ denote the group of continuous functions from a topological space $X$ into a topological group $G$ with the pointwise multiplication and the compact-open topology. We show that there is a natural topology on the collection of normal subgroups $\Delta(X)$ of $C(X, G)$ of the $M_{p}=\{f \in C(X, G): f(p)=e\}$ which is analogous to the hull-kernel topology on the commutative Banach algegra $C(X)$ of all continuous real or complex-valued functions on $\mathrm{X}$. We also investigate homomorphisms between groups $\mathrm{C}(\mathrm{X}, \mathrm{G})$ and $\mathrm{C}(\mathrm{Y}, \mathrm{G})$.

KEY WORDS AND PHRASES. Continuous functions, topological group, compact-open 'topology, hull-kernel topology, normal subgroups, S-pair, S-topology, Banach algebra, structure space.

1980 MATHEMATICS SUBJECT CLASSIFICATION CODES: Primary 54C35, 54H10, Secondary 22A05, $20 F 30$.

1. INTRODUCTION AND NOTATION.

Suppose $X$ is a compact topological space and suppose $C(X)$ is the algebra of 
all continuous real or complex-valued functions on $\mathrm{X}$ with the usual pointwise operations and the supremum norm. Then $\mathrm{C}(\mathrm{X})$ is a regular commutative Banach algebra with identity and $X$ is homeomorphic to the maximal ideal space $\Delta(C(X))$ of the algebra $C(X)$, where $\Delta(C(X))$ is endowed with the Gel'fand topology which coincides with the hull-kernel topology since $C(X)$ is regular, [3]. If $X$ is a topological space and $G$ is a topological group, let $C(X, G)$ be the topological group of all continuous functions from $X$ into $G$ under pointwise multiplication and the compact-open topology. In Section 2 of this paper, we study spaces of normal subgroups of $C(X, G)$. There is a natural topology, analogous to the hull-kernel topology in Banach algebra, for the collection of normal subgroups of the form $M_{p}=M_{p}(X, G)=\{f \in C(X, G): f(p)=e\}$, where $e$ is the identity element of $G$; the resulting topological space will be denoted by $\Delta(X)$. We show that, with some mild restriction on $X$ and $G, X$ is homeomorphic to $\Delta(X)$, and that $\Delta\left(X^{*}\right)$ is the onepoint compactification of $\Delta(X)$, where $X^{*}$ is the one-point compactification of the locally compact space $\mathrm{X}$. Some theorems on homomorphisms and extension of homomorphisms in $C(X, G)$ are considered in Section 3 . We also prove a correct version of a theorem originally stated in [7, theorem 8].

All spaces considered in this paper are assumed to be Hausdorff unless specified. For topological spaces $X$ and $Y$, the function space $F \subset C(X, Y)$ is understood to be endowed with the compact-open topology whenever it is referred to topologically. $I_{0}(X, G)$, or simply $I_{0}$ if no confusion should occur, will denote the identity element of the group $C(X, G)$.

\section{THE STRUCTURE SPACES.}

For a topological space $X$ and a topologịcal group $G$, let $F=C(X, G)$. If $X$ is compact and $G$ is a Lie group, then $\Gamma$ and $M_{p}, p \in X$, are in general $\ell_{2}$ manifolds (c.f. [1]). It is easy to see that $M_{a}$ is locally contractible at $I_{0}$, where $a \in X$, if $X$ is a locally compact group locally contractible at a, and that 
every $M_{p}, p \in X$, is $n$-simple for every positive integer $n$ if $X$ is a locally compact contractible space. It is also easy to see that the topological group $\Gamma$ is a group with equal left and right uniformities if so is the group G, and that, if $G$ is the projective limit of the inverse system of topological groups $\left\{\left(G_{\alpha}, f_{B \alpha}\right): \alpha, \beta \in A\right\}$, then $M_{p}$ is the projective limit of the inverse system $\left\{\left(M_{p}\left(X, G_{\alpha}\right), f_{B \alpha}^{p}\right): \alpha, \beta \in A\right\}$, where $f_{B \alpha}^{p}(f)=f_{B \alpha} \circ f$ for every $f \in M_{p}(X, G)$.

Throughout this paper the spaces $X$ and $G$ will be subject to the following condition.

DEFINITION 1 [6]. A pair $(X, G)$ of a topological space $X$ and a topological group $G$ is called an S-pair if for each closed subset $A$ of $X$ and $x \notin A$, there exists $f \in \Gamma$ such that $f(x) \neq e$ and $Z(f)=\{x: f(x)=e\} \supset A$.

It is clear that $(X, G)$ is an S-pair if $X$ is completely regular and $G$ is path connected or if $\mathrm{X}$ is zero-dimensional. It is also clear that $\mathrm{X}$ is completely regular if $(X, G)$ is an S-pair, and that $\left(\underset{\alpha \in A}{X_{a}}, \underset{\alpha \in A}{\pi} G_{a}\right)$ is also an S-pair whenever $\left(X_{\alpha}, G_{\alpha}\right)$ is an S-pair for each $\alpha \in A$. Magill called a space X a V-space, [4], if for points $p, q, x$, and $y$ of $x$, where $p \neq q$, there exists a continuous function $f$ of $x$ into itself such that $f(p) \neq x$ and $f(q) \neq y$, and has shown that every completely regular path connected space and every zero-dimensional space is a Vspace. It is easy to see that $\left(\operatorname{maA}_{\alpha \in A} X_{a}, G\right)$ is an S-pair if each $\left(X_{a}, G\right), a \in A$, is is an S-pair and if $G$ is a V-space. If $G$ is a topological group such that $(G, G)$ is an S-pair, $G$ may not be a V-space. For example, let $G_{1}$ be the additive group of real numbers with the usual topology and let $G_{2}$ be any non-trivial finite group with the discrete topology, then $\left(G_{1} \times G_{2}, G_{1} \times G_{2}\right)$ is an S-pair since $\left(G_{1}, G_{1}\right)$ and $\left(G_{2}, G_{2}\right)$ are S-pairs. Since the topological group $G_{1} \times G_{2}$ is not connected with the identity component isomorphic to $G_{1}, G_{1} \times G_{2}$ is not a V-space as it follows from [4, Theorem 3.5]. It is pointed out in [7] that $X$ is hemicompact and $G$ is metrizable if $(X, G)$ is an S-pair, $G$ is a V-space, and $\Gamma$ is first countable. 
It is well-known (c.f. [2]) that, for every topological space $X$, there exists a completely regular space $Y$ such that $C(Y)$ is (algebraically) isomorphic to $C(X)$, where $C(Z)$ is the ring of continuous real-valued function on the space $Z$. Using the similar argument mutatis mutandis as used in the construction of the space $Y$, it is a straightforward to see that, for every topological space $X$ and a topological group $G$, there is a completely regular space $Y_{G}$ such that $C\left(Y_{G}, G\right)$ is continuously isomorphic to $C(X, G)$, and that, in the case $G$ is path connected, $\left(Y_{G}, G\right)$ is an S-pair and the associated space $Y_{G}$ is independent of the group $G$ within the category of path connected topological groups. The latter means that $Y_{G_{1}}=Y_{G_{2}}$ whenever $G_{1}$ and $G_{2}$ are path connected groups. It follows from the construction of the space $Y_{G}$ that $X=Y_{G}$ if $(X, G)$ is an S-pair.

Because of the remarks just made above, we shall now assume that $(X, G)$ is an S-pair.

For a collection $\sum$ of normal subgroups of $\Gamma=C(X, G)$, we define "*" as follows: If $U \subset \sum$ and $U \neq \phi$, let $U^{*}=\left\{M \in[: M \supset\right.$ nU $\}$, let $\phi^{*}=\phi$.

THEOREM 1. "*" is a closure operator on $\sum$ if and only if whenever $M \in \sum$ and $M \supset M_{1} \cap M_{2}$, where $M_{1}$ and $M_{2}$ are intersections of some subsets of $\sum$, then either $M \supset M_{1}$ of $M \supset M_{2}$.

PROOF: It is clear that $U^{*} \supset U,\left(U^{*}\right) *=U^{*}, \phi^{*}=\phi$, and that $U^{*} \cup V^{*} \subset(U \cup V) *$ for subsets $U$ and $V$ of $\sum$. Hence "*" is a closure operator if and only if $U * U V * \supset(U \cup V) *$ for subsets $U$ and $V$ of $\sum$. Now if $M_{1}=n U$, and $M_{2}=n V$, then $(U \cap V) *=\left\{M \in\left[: M \supset M_{1} \cap M_{2}\right\}\right.$. Hence we have the theorem.

DEFINITION 2. If "*" is a closure operator on $\sum$, we shall refer the resulting topology, not necessarily Hausdorff, on $\sum$ as the S-topology, and the resulting space will be referred to as a G-structure space, or simply structure space, of the space $x$.

COROLLARY: If $\sum$ admits the S-topology, so is every subset of $\mathcal{E}$. 
REMARK 2. If $G$ is path connected, we may speak of structure spaces for the space $X$ without referring to the group since $C(X, G)$ and $C(X, R)$ are isomorphic in this case.

LEMMA 3. If a collection of normal subgroup $\sum$ of $\Gamma$ admits the S-topology, then a subset $A$ of $\sum$ is closed if and only if there exists a normal subgroup $M_{0}$ of $\Gamma$ which is the intersection of some subset of $\sum$ such that

$A=\left\{M \in\left[: M \supset M_{0}\right\}\right.$. In fact, $M_{0}=n A$.

PROOF: Suppose $A \subset \sum$ is closed, then $A=\bar{A}=\left\{M \in\left[: M \supset \cap A=M_{0}\right\}\right.$.

Conversely, suppose that there exists a normal subgroup $M_{0}$ of $\Gamma$, where $M_{0}=n U$ for some $U \subset \sum$, such that $A=\left\{M \in\left[: M \supset M_{0}\right\}\right.$. Then $\bar{A}=\{M \in[: M \supset \cap A\}=A$. Hence $A$ is closed.

THEOREM 4. If a collection of normal subgroups $\sum$ of $\Gamma$ admits the S-topology, then $\left[\right.$ is Hausdorff if and only if for $M_{1}, M_{2} \in \sum, M_{1} \neq M_{2}$, there are $I_{1}$ and $I_{2}$, where $I_{1}=n U_{1}, I_{2}=n U_{2}$ and $U_{1}, U_{2} \subset \sum$, such that $M_{1} \supset I_{1}, M_{2} \supset I_{2}, M_{1} \not \supset I_{2}$, $M_{2} \not \supset I_{1}$, and $I_{1} \cap I_{2}=n \sum$.

PROOF: Suppose that $\sum$ is Hausdorff, and let $M_{1}, M_{2} \in \sum, M_{1} \neq M_{2}$. Thus there are disjoint open sets $U_{1}$ and $U_{2}$ in $\left[\right.$ such that $M_{1} \subset U_{1}$, and $M_{2} \subset U_{2}$. If $A_{1}=$ $\sum-U_{2}, A_{2}=\sum-U_{1}$, then $A_{1}$ and $A_{2}$ are closed and $M_{1} \in A_{1}, M_{2} \in A_{2}$. Using Lemma 3 , we have $A_{i}=\left\{M \in\left[: M \supset \cap A_{i}\right\}, i=1,2\right.$. If we let $I_{i}=n A_{i}, i=1,2$, then $M_{1} \supset I_{1}, M_{2} \supset I_{2}, M_{1} \not \supset I_{2}, M_{2} \not I_{1}$ and $I_{1} \cap I_{2}=n \sum$.

Conversely, assume that the stated property holds, and let $M_{1}, M_{2} \in \sum$ such that $M_{1} \neq M_{2}$. Then there are subsets $U_{1}$ and $U_{2}$ of $\sum$ such that if $I_{i}=n U_{i}$, $i=1,2, M_{1} \supset I_{1}, M_{2} \supset I_{2}, M_{1} \not I_{2}, M_{2} \not \supset I_{1}$, and $I_{1} \cap I_{2}=n\left[\right.$. Let $B_{1}=$ $\left\{M \in\left[: M \supset I_{i}\right\}, i=1,2\right.$. Then $B_{i}$ are closed by Lemma $3, M_{1} \in B_{1}, M_{2} \in B_{2}$, $M_{1} \notin B_{2}$ and $M_{2} \notin B_{1}$. If we let $v_{2}=\sum-B_{1}, v_{1}=\sum-B_{2}$, then $M_{1} \in v_{1}, M_{2} \in v_{2}$, and $v_{1} \cap v_{2}=\phi$. To see that $v_{1} \cap v_{2}=\phi$, it suffices to show that if $M \in \sum$, then either $M \in B_{1}$ or $M \in B_{2}$. Now $M \in \sum$ implies $M \supset \cap \sum=I_{1} \cap I_{2}$. This means 
that either $M \supset I_{1}$ or $M \supset I_{2}$ since $\sum$ admits the $S$-topology. Hence $M \in B_{1}$ or $M \in B_{2}$. This completes the proof.

If we denote by $\Delta(X)$ the collection of all normal subgroups of $\Gamma$ of the form $M_{p}=\{f \in C(X, G): f(p)=e\}, p \in X$, then the following theorem states that $\Delta(X)$ admits the S-topology and that the S-topology is Hausdorff if $(X, G)$ is an S-pair.

THEOREM 5. $\Delta(\mathrm{X})$ admits the Hausdorff S-topology.

PROOF: Let $U$ and $V$ be subsets of $\Delta(X)$, and let $O_{1}=\left\{P \in X: M_{p} \in U\right\}$ and $0_{2}=\left\{q \in X: M_{q} \in V\right\}$. It is, by Theorem 1, sufficient to show that, if $\mathrm{M}_{\mathrm{q}} \supset\left(\underset{\mathrm{p} \in \mathrm{O}_{1}}{n} \mathrm{M}_{\mathrm{p}}\right) \cap\left(\underset{\mathrm{k} \in \mathrm{O}_{2}}{n} \mathrm{M}_{\mathrm{k}}\right)$, then either $\mathrm{M}_{\mathrm{q}} \supset \underset{\mathrm{p} \in \mathrm{O}_{1}}{n} \mathrm{M}_{\mathrm{p}}$ and $\mathrm{M}_{\mathrm{q}} \supset \underset{\mathrm{k} \in \mathrm{O}_{2}}{n} \mathrm{M}_{k} \cdot$ Suppose otherwise, then there exist $f \in \underset{p \in O_{1}}{n} M_{p}-M_{q}$ and $g \in \underset{k \in O_{2}}{M_{k}}-M_{q}$. This implies that $\mathrm{q} \notin \overline{\mathrm{o}}_{1}$ and $\mathrm{q} \notin \overline{\mathrm{o}}_{2}$. For if $\mathrm{q} \in \overline{\mathrm{o}}_{1}$, then there is a net $\left\{\mathrm{q}_{\alpha}\right\}$ in $o_{1}$ such that $\mathrm{q}_{\alpha} \rightarrow \mathrm{q}$. Then $f(q) \rightarrow f(q)$, and hence $f(q)=$ e since $f(q)=$ e for each $\alpha$. Similarly, $\mathrm{q} \notin \overline{0}_{2}$. Hence $\mathrm{q} \notin \overline{\mathrm{O}_{1} \cup \mathrm{O}_{2}}$. But $(X, G)$ is an $\mathrm{S}=$ pair, let $\mathrm{h} \in \Gamma$ such that $\overline{0_{1} \cup O_{2}} \subset \mathrm{Z}(\mathrm{h})$ but $\mathrm{h}(\mathrm{q}) \neq \mathrm{e}$. This would show that $\mathrm{h} \in\left(\underset{\mathrm{p} \in \mathrm{O}_{1}}{\mathrm{M}}{ }_{\mathrm{p}}\right) \cap\left(\underset{\mathrm{k} \in \mathrm{O}_{2}}{\mathrm{M}} \mathrm{M}_{\mathrm{k}}\right)$ but $h \notin M_{q}$, a contradiction. Hence either $\left.M_{q} \supset \underset{p \in O_{1}}{M_{p}}\right)$ or $M_{q} \supset\left(\underset{k \in O_{2}}{n} M_{k}\right)$, and $\Delta(X)$ admits the S-topology.

Next to show that the S-topology is Hausdorff. Let $M_{p}, M_{q} \in \Delta(X)$, where $\mathrm{p} \neq \mathrm{q}$. Since $\mathrm{X}$ is $\mathrm{T}_{2}$, let $\mathrm{o}_{1}$ and $\mathrm{o}_{2}$ be open sets in $\mathrm{X}$ such that $\mathrm{P} \in \mathrm{o}_{1}, q \in 0_{2}$ and $o_{1} \cap o_{2}=\phi$. If $c_{2}=x-o_{1}$ and $c_{1}=x-o_{2}$, then $p \in C_{1}$ and $q \in C_{2}$. If $I_{1}=\underset{k \in C_{1}}{n} M_{k}$ and $I_{2}=\underset{k \in O_{2}}{n} M_{k}$, then $I_{1} \cap I_{2}=n \Delta(X)$ since $C_{1} \cup C_{2}=X, M_{p} \supset I_{1}$, and $M_{q} \supset I_{2}$. To see that $M_{p} \not \supset I_{2}$ note that $p \notin C_{2}$, hence there exists $f \in \Gamma$ such that $F\left(C_{2}\right)=$ e but $f(p) \neq$ e. Thus $f \in \underset{k \in C_{2}}{n} M_{k}$ but $f \notin M_{p}$. This shows that $M_{p} \not f I_{2}$. Similarly, we have $M_{q} \not \supset I_{1}$. This completes the proof that $\Delta(X)$ is $T_{2}$, by Theorem 4 .

Note that the S-topology defined above for $\Delta(X)$ is analogous to the hullkernel topology, which coincides with the Gel'fand topology, on the maximal ideal space of the commutative Banach algebra $C(X)$. 
For each $\alpha \in I$, let $A_{\alpha}$ be a closed set of a structure space $\sum$. Then, by Lemma 3, there exists a normal subgroup $M_{\alpha}$ of $\Gamma$ which is the intersection of some subset of $\sum$ such that $A_{\alpha}=\left\{M \in\left[: M \supset M_{\alpha}\right\}\right.$. If we denoted by $\left[\underset{\alpha \in I}{u} M_{\alpha}\right]$ the normal subgroup of $\Gamma$ generated by $\underset{\alpha \in I}{U} M_{\alpha}$, then we have the following lemana whose proof is straightforward and hence omitted.

LEMMA 6. $\underset{\alpha \in I}{n A_{\alpha}}=\{M \in[: M \supset[\underset{\alpha \in I}{U} M]\}$

THEOREM 7. A structure space $\sum$ of $X$ is compact if and only if every collection of normal subgroups $\left\{\mathrm{N}_{\alpha}\right\}_{\alpha \in \mathrm{I}}$ of $\Gamma$, each of which is the intersection of some subset of $\left[\right.$, such that $\left[\cup_{\alpha \in I} N_{\alpha}\right] \notin M$ for each $M \in \sum$ has a finite subcollection. $\left\{N_{\alpha_{1}}, N_{\alpha_{2}}, \ldots, N_{\alpha_{n}}\right\}$ such that $[\underbrace{n}_{i=1} N_{\alpha_{i}}] \notin M$ for each $M \in \sum$.

PROOF : Suppose $\left[\right.$ is compact, and let $\left\{\mathrm{N}_{\alpha}\right\}_{\alpha \in I}$ be a collection of normal subgroups of $\Gamma$, each of which is the intersection of some subset of $\sum$, such that

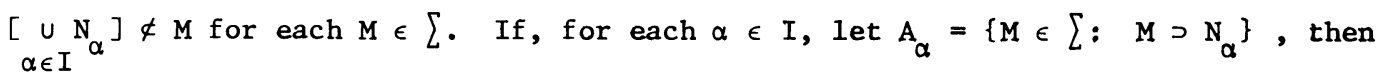
$A_{\alpha}$ is closed in $\left[\right.$, Lemma 3 , and $\underset{\alpha \in I}{n} A_{\alpha}=\left\{M \in\left[: M \supset \underset{\alpha \in I}{\left.\left[\cup_{n} N_{\alpha}\right]\right\}}=\phi\right.\right.$. Hence, by the compactness of $\sum$, there exist $\alpha_{1}, \alpha_{2}, \ldots, \alpha_{n}$ such that $\prod_{i=1}^{n} A_{\alpha_{i}}=\phi$; i.e., there exist $\alpha_{1}, \alpha_{2}, \ldots, \alpha_{n}$ such that $\left\{M \in\left[: M \supset\left[\bigcup_{i=1}^{n} N_{\alpha_{i}}\right]\right\} \stackrel{i=1}{=} \phi\right.$. Hence $[\underbrace{n}_{i=1} N_{\alpha_{i}}] \notin M$ for each $M \in \sum$.

Conversely, suppose that $\sum$ has the stated property, and let $\left\{A_{\alpha}\right\}_{\alpha \in I}$ be a collection of closed sets with the finite intersection property, where $A_{\alpha}=$ $\left\{M \in\left[: M \supset N_{\alpha}\right\}\right.$ and $N$ is the intersection of some subset of $\sum$. Suppose that $n_{\alpha \in I} A_{\alpha}=\phi . \quad$ Then $\left\{M \in\left[: M \supset\left[\underset{\alpha \in I}{\cup N_{\alpha}}\right]=\phi\right.\right.$, hence $\left[\underset{\alpha \in I}{u N_{\alpha}}\right] \notin M$ for each $M \in \sum$. Thus, by the hypothesis, there exist $\alpha_{1}, \alpha_{2}, \ldots, \alpha_{n}$ such that $\left[\bigcup_{i=1}^{n} N_{\alpha_{i}}\right] \not M$ for each $M \in \sum$. This would imply that $\prod_{i=1}^{n} A_{\alpha_{i}}=\phi$, a contradiction. Hence $\sum$ is compact.

COROLLARY. A structure space $\sum$ of $X$ is compact if every normal subgroup $N$ of $\Gamma$ not contained in any element of $\sum$ contains a finitely generated normal sub- 
group of $\mathrm{N}$ not contained in any element of $\lceil$.

PROOR: Assume that the stated property holds in $\left[\right.$, and 1 et $\left\{\mathrm{N}_{\alpha}\right\}_{\alpha \in \mathrm{I}}$ be a collection of normal subgroups of $\Gamma$, each of which is the intersection of some subset of $\sum$, such that $\left[\underset{\alpha \in I}{U} N_{\alpha}\right] \not M$ for every $M$ in $\left[\right.$. Let $N=\left[\underset{\alpha \in I}{U N_{\alpha}}\right]$. Then $N \notin M$ for every $M$ in $\sum$, thus $N$ contains a finitely generated normal subgroup $B$ such that $B \not M$ for each $M \in \sum$. Let $B=\left[a_{\alpha_{1}}, a_{\alpha_{2}}, \ldots, a_{\alpha_{n}}\right]$, where $a_{\alpha_{i}} \in N_{\alpha_{i}}$, $i=1,2, \ldots, n$. Then $\left[\bigcup_{i=1}^{n} N_{\alpha_{i}}\right] \notin M$ for every $M$ in $\sum$. Hence $\sum$ is compact by Theorem 7 .

We shall call a normal subgroup $N$ of $\Gamma$ free if there is no $p \in X$ such that $f(p)=e$ for each $f \in N$.

COROLLARY. $\Delta(\mathrm{X})$ is compact if every free normal subgroup $\mathrm{N}$ of $\mathrm{T}$ contains a finitely generated free normal subgroup.

THEOREM 8. The mapping $\psi: X \rightarrow \Delta(X)$ defined by $\psi(x)=M_{x}, x \in X$, is a homeomorphism.

PROOF: Clearly, $\psi$ is one-to-one and onto.

For the continuity of $\psi$, let $A \subset \Delta(X)$ be closed. Then there exists a normal subgroup $M_{0}$ of $\Gamma$ such that $A=\left\{M_{X} \in \Delta(X): M_{X} \supset M_{0}\right\}$. We shall see that $\psi^{-1}(A)$ is closed. For this purpose, let $\left\{x_{\alpha}\right\}$ be a net in $\psi^{-1}(A)$ converging to $x \in X$. Then $M_{x_{\alpha}} \supset M_{0}$ for each $\alpha$. If $M_{x} \not M_{0}$, there exists $f \in M_{0}-M_{x}$ which would imply that $f(x) \neq e$, a contradiction since $f\left(x_{\alpha}\right)+f(x)$ and $f(x)=$ e for each $\alpha$.

Next to show that $\psi$ is a closed map. Let $C$ be closed in $X$, and let $M_{0}=\underset{x \in C}{n} M_{X}$. We claim that $\psi(C)=\left\{M_{X} \in \Delta(X): M_{X} \supset M_{0}\right\}$, which would imply that $\psi(C)$ is closed. It is clear that $\psi(C) \subset\left\{M_{X} \in \Delta(X): M_{X} \supset M_{0}\right\}$. Now let $M_{X} \in\left\{M_{X} \in \Delta(X): M_{X} \supset M_{0}\right\}$. Then $M_{X} \supset M_{0}$. Suppose $x \notin C$, then there exists $f \in C(X, G)$ such that $f(C)=e$ but $f(x) \neq$ e. Hence $f \in M_{0}$ but $f \notin M_{x}$, a contradiction. Thus $x \in C$, and we have $M_{x} \in \psi(C)$.

If $A$ is a commutative Banach algebra without identity, and if $A(e)$ is the algebra obtained by adjoining an identity to $A$, then the maximal ideal space 
$\Delta(A(e))$, with the Gel'fand topology, is the one-point compactification of $\Delta(A)$. Using the previous results, we can also state the following theorem whose proof is now trivial.

THEOREM 9. If $X$ is a locally compact space and $X *$ its one-point compactification, then $\Delta\left(X^{*}\right)$ is the one-point compactification of $\Delta(X)$, and $\Delta\left(X^{*}\right)=$ $\Delta(X) \cup\left\{M_{\infty}\right\}$, where $M_{\infty}=\left\{f \in C\left(X^{*}, G\right): f(\infty)=e\right\}$.

3. HOMOMORPHISMS OF $\mathrm{C}(\mathrm{X}, \mathrm{G})$.

In this section, we shall study homomorphisms of the group $\mathrm{C}(\mathrm{X}, \mathrm{G})$ into the group $\mathrm{C}(\mathrm{Y}, \mathrm{G})$ which leads us to have another version of a theorem originally announced in [7]. We shall also, at the end of the section, consider extensions of homomorphisms of the group $C(X, G)$. All pairs $(Z, G)$ are again assumed to be S-pairs.

DEFINITION 3. (1) A homomorphism $\phi$ of the group $C(Y, G)$ into the group $C(X, G)$ is said to be a constant-preserving if $\phi$ maps every constant function on $\mathrm{Y}$ into the corresponding constant function on $\mathrm{X}$.

(2) A homomorphism $\phi$ of the group $C(Y, G)$ into the group $C(X, G)$ which has the property that $\phi^{-1}(\Delta(\mathrm{X})) \subset \Delta(\mathrm{Y})$ is called an F-homomorphism.

It is easy to construct an example of a homomorphism $\phi: C(Y, G) \rightarrow C(X, G)$ which is an F-homomorphism but is not constant-preserving. The following example [5], shows that the converse does not hold either.

EXAMPLE. Let $\mathrm{Y}=[0,1]$ be the closed unit interval, and let $\mathrm{X}=$ $([-1,1] \times\{0\}) \cup(\{0\} \times(0,1])$ considered as a subspace of $\mathrm{R}^{2}$. For each $f \in C(Y, R)$, define $\phi(f) \in C(X, R)$ by

$$
\begin{aligned}
& \phi(f)(t, 0)=f\left(\frac{1}{4}(t+1)\right), \quad t \in[-1,1] \\
& \phi(f)(0, s)=f\left(\frac{1}{2}(s+1)\right)+f\left(\frac{1}{2}\right), s \in[0,1]
\end{aligned}
$$

Then $\phi$ is a constant-preserving isomorphism of $C(Y, R)$ onto $C(X, R)$. If $g \in C(X, R)$, 
then

$$
\phi^{-1}(g)(y)= \begin{cases}g(4 y-1,0) & y \in\left[0, \frac{1}{2}\right] \\ g(0,2 y-1)+g(1,0)-g(0,0), & y \in\left[\frac{1}{2}, 1\right]\end{cases}
$$

Now choose $g \in C(X, R)$ such that $Z(g)=\left\{\left(0, \frac{1}{2}\right)\right\}$ and that $g(1,0)-g(0,0)>0$, then $g \in M_{\left(0, \frac{1}{2}\right)}$, but $Z\left(\phi^{-1}(g)\right)=\phi$. Hence $\phi$ is not an F-homomorphism.

THEOREM 10. Suppose that $\phi: C(Y, G) \rightarrow C(X, G)$ is a continuous constant-preserving F-homomorphism of $C(Y, G)$ into $C(X, G)$. Then

(1) $\phi$ induces a one-to-one continuous map of $\Delta(\mathrm{X})$ into $\Delta(\mathrm{Y})$, and

(2) $\phi$ induces a continuous map $j$ of $X$ into $Y$ such that $j(x)=y$ if and only if $\phi(g)(x)=g(y)$ for each $g \in C(Y, G)$.

PROOF. (1) for each $x \in X$, let $h x: C(X, G) \rightarrow G$ be the evaluation map defined by $h x(f)=f(x), f \in C(X, G)$, and let $M_{x}=$ kerhx. Define $h(x): C(Y, G) \rightarrow G$ by $h(x)=h x \circ \phi, x \in X$. Then $\operatorname{ker} h(x)=\phi^{-1}\left(M_{x}\right)$, hence $\operatorname{ker} h(x)=M_{y}$ for some $\mathrm{y} \in \mathrm{Y}$. Such an $\mathrm{y}$ is unique and we have $\mathrm{h}(\mathrm{x})=\mathrm{hy}$. Now we define a mapping

$\Phi: \quad(X) \rightarrow \Delta(Y)$ by $\Phi\left(M_{X}\right)=M_{y}$.

Clearly $\Phi$ is one-to-one. For the continuity of $\Phi$, let $\mathrm{A}=$ $\left\{M_{y} \in \Delta(Y): M_{y} \supset M_{1}\right\}$, where $M_{1}$ is the intersection of some subset $U$ of $\Delta(Y)$, be a closed set in $\Delta(Y)$. We claim that $\Phi^{-1}(A)=\left\{M_{X} \in \Delta(X): M_{X} \supset \phi\left(M_{1}\right)\right\}$ and that $\phi\left(M_{1}\right)$ is the intersection of the subset $\phi(U)$ of $\Delta(X)$. In fact, let $M_{X} \supset \phi\left(M_{1}\right)$. Then $\phi^{-1}\left(M_{x}\right) \supset M_{1}$. If $\Phi\left(M_{x}\right)=M_{y}, M_{y}=\operatorname{ker} h y=\operatorname{ker}(h x \circ \phi)=\phi^{-1}\left(M_{x} \supset M_{1}\right.$, hence $M_{y} \in A$, thus $M_{x}=\Phi^{-1}\left(M_{y}\right) \in \Phi^{-1}(A)$. Conversely, let $M_{z} \in \Phi^{-1}(A)$. Then $\Phi\left(M_{z}\right) \in A$. If $\Phi\left(M_{z}\right)=M_{y} \in A$ for some $y \in Y$, then $H y=h z \circ \phi$, hence $\phi\left(M_{1}\right) \subset M_{z}$. It is easy to see that $\phi\left(M_{1}\right)$ is the intersection of the subset $\phi(U)$ of $\Delta(X)$. Therefore $\Phi^{-1}(\mathrm{~A})$ is closed in $\Delta(\mathrm{X})$, and $\Phi$ is continuous.

(2) Let the mapping $j: X \rightarrow Y$ be defined by $j=\psi_{y}^{-1} \circ \Phi \circ \psi x$, where 
$\psi_{z}: Z \rightarrow \Delta(Z)$ is the mapping of Theorem 8. Then clearly $j$ is continuous and $j(x)=y$ if and only if $\Phi\left(M_{x}\right)=M_{y}$. To see that $j(x)=y$ if and only if $\phi(g)(x)=g(y)$ for every $g \in C(Y, G)$, let $j(x)=y \cdot$ Then $\Phi\left(M_{x}\right)=M_{y}$. Thus $\operatorname{ker}(h x \circ \phi)=M_{y}$. Let $g \in C(Y, G)$. If $g \in M_{y}, h x \circ \phi(g)=e$, and we have $\phi(g)(x)=g(y)$. If $g \notin M_{y}$, there exists $c \in G$ such that $g \in \underline{c} M_{y}$, where $\underline{c}$ is the constant mapping of $\mathrm{X}$ into $\mathrm{c}$, hence $\mathrm{g}=\underline{\mathrm{c} k}$ for some $\mathrm{k} \in \mathrm{My}$. Now $\mathrm{hx} \circ \phi(\mathrm{g})=$ $h x \circ \phi(\underline{c k})=h x(\underline{c} \phi(k))=c \phi(k)(x)=c$, while $g(y)=c k(y)=c$. Hence $\phi(g)(x)=g(y)$ for each $g \in C(Y, G)$. Conversely, if $\phi(g)(x)=g(y)$ for each $g \in C(Y, G)$, then, for $g \in C(Y, G), h x \circ \phi(g)=\phi(g)(x)=g(y)=h y(g)$. Thus $\Phi(M x)=M y$, and we have that $j(x)=y$.

REMARK: It is easy to see that, if the mapping $\phi$ in Theorem 10 is an onto map, then $\Phi$ is an embedding.

THEOREM 11. A continuous homomorphism $\phi$ of $C(Y, G)$ into $C(X, G)$ is a constant-preserving F-homomorphism if and only if there exists $f \in C(X, Y)$ such that $\phi(k)=k \circ f$ for every $k \in C(Y, G)$.

PROOF : It is clear that a homomorphism $\phi$ of the form $\phi(k)=k$ of for every $k \in C(Y, G)$ is a constant-preserving $F$-homomorphism. Conversely, if $\phi$ is a constant-preserving F-homomorphism, and if $\mathrm{j}$ is the continuous map of $\mathrm{X}$ into $\mathrm{Y}$ as defined in Theorem 10, then, for each $k \in C(Y, G), \phi(k)(x)=k(y)=k \circ j(x)$, where $\mathrm{j}(\mathrm{x})=\mathrm{y}$, Hence $\phi(\mathrm{k})=\mathrm{k} \circ \mathrm{j}$ for each $\mathrm{k} \in \mathrm{C}(\mathrm{Y}, \mathrm{G})$.

COROLLARY. A homomorphism $\phi$ of $C(Y, G)$ int'o $X(X, G)$ is a constant-preserving F-homomorphism if and only if there exists $f \in C(X, Y)$ such that $\phi(k)=k \circ f$ for every $k \in C(Y, G)$.

PROOF: Note that the group topologies for $C(Y, G)$ and $C(X, G)$ are not relevant in the proof of Theorem 10. Hence take discrete topologies for the groups $C(Y, G)$ and $C(X, G)$, then apply the proof of Theorem 11 .

As a consequence of the discussions made above, we can now state a correct 
version of the theorem originally stated in [7, Theorem 8] in the following.

THEOREM 12. If there exists an isomorphism $\phi$ between groups $C(Y, G)$ and $C(X, G)$ which is constant-preserving such that both $\phi$ and $\phi^{-1}$ are F-homomorphisms, then $\mathrm{X}$ and $\mathrm{Y}$ are homeomorphic.

PROOF: It is clear that $\phi^{-1}$ is also constant-preserving if $\phi$ is. Applying the above corollary to $\phi$ and $\phi^{-1}$, there exist functions $\mathrm{j} \in \mathrm{C}(\mathrm{X}, \mathrm{Y})$ and $\ell \in \mathrm{C}(\mathrm{Y}, \mathrm{X})$ such that $\phi(k)=k \circ j$ for each $k \in C(Y, G)$ and $\phi^{-1}(k)=k \circ \ell$ for each $k \in C(X, G)$. Consequently, we have that $\ell \circ j(x)=x$ and $j \circ \ell(y)=y$ for $x \in X$ and $y \in Y$. To see this suppose that there exists $x \in X$ such that $\ell \circ j(x) \neq x$, then we have $f \in C(X, G)$ such that $f(l \circ j(x)) \neq f(x)$ or $f \circ \ell \circ j(x) \neq f(x)$. Hence $\left(\phi^{-1}(f) \circ j\right)(x) \neq f(x)$, and thus $\phi\left(\phi^{-1}(f)\right)(x) \neq f(x)$ which leads to $f(x) \neq f(x)$. Similarly, $\mathrm{j} \circ \ell(\mathrm{y})=\mathrm{y}$. Hence $\mathrm{j}$ is a homeomorphism of $\mathrm{X}$ onto $\mathrm{Y}$.

For topological spaces $\mathrm{X}$ and $\mathrm{Y}$, it is clear that the space $\mathrm{C}(\mathrm{X}, \mathrm{Y})$ may be embedded into the space $\mathrm{C}(\mathrm{X} \times \mathrm{Z}, \mathrm{Y})$ as a retract for any space $\mathrm{Z}$, and that every homomorphism of the topological group $C(X, G)$ into a topological group L may be extended to a homomorphism of the topological group $C(X \times Y, G)$ into $L$ for any topological group L. We shall conclude this paper with the following result concerning an extension of F-homomorphisms.

THEOREM 13. Suppose $A$ is a closed subset of $X$. Then every constant-preserving F-homomorphism $h$ of the topological group $C(G, G)$ into the topological group $C(A, G)$ may be extended to a homomorphism $H$ of the same kind from the topological group $C(G, G)$ into the topological group $C(X, G)$ such that $I \circ H=h$ if every continuous function $f: A \rightarrow G$ may be continuously extended to all of $x$, where $I: C(X, G) \rightarrow C(A, G)$ be the map defined by $I(f)=f \circ i$ for $f \in C(X, G)$, $i$ being the inclusion map of $A$ into $x$.

PROOF: For necessity, let $f$ : $A \quad G$ be any continuous function, and let $f *$ : $C(G, G) \quad C(A, G)$ be the natural homomorphism induced by $f$, namely $f *(k)=$ 
$k$ - $f$ for each $k \in C(G, G)$. Then $f *$ is a constant-preserving $F$-homomorphism, by Theorem 11. Hence there exists a constant-preserving F-homomorphism $H$ of the topological group $C(G, G)$ into $C(X, G)$ such that $I \circ H=f *$. Let $\theta \in C(X, G)$ such that $H(k)=k \circ \Theta$ for every $k \in C(G, G)$. If $i_{d}$ denotes the identity map of $G$ into itself, then, for $a \in A, \theta(a)=\left(i_{d} \circ \theta\right)(a)=H\left(i_{d}\right)(a)=H\left(i_{d}\right)(i(a))=H\left(i_{d}\right)$ $\circ i(a)-I\left(H\left(i_{d}\right)\right)(a)=(I \circ H)\left(i_{d}\right)(a)=f *\left(i_{d}\right)(a)\left(i_{d} \circ f\right)(a)=f(a)$. Hence $\theta$ is an extension of $f$ to all of $X$.

For sufficiency, assume that every continuous function $f: A \rightarrow G$ may be extended continuously to all of $X$, and let $h: C(G, G) \rightarrow C(A, G)$ be a constant-preserving homomorphism of the topological group $C(G, G)$ into the topological group $C(A, G)$. Then there exists $f \in C(A, G)$ such that $h(k)=k \circ f$ for every $k \in C(G, G)$. If we denote by $f$ the extension of $\hat{f}$ to all of $x$, define a function $H$ : $C(G, G) \rightarrow C(X, G)$ by $H(k)=k \circ \hat{f}$ for each $k \in C(G, G)$. Then $H$ is a constant-preserving F-homomorphism and $I \circ H=h$. This completes the proof.

In particular, if $\mathrm{X}$ is a normal space, and $\mathrm{A}$ a closed subset of $\mathrm{X}$, then every constant-preserving $F$-homomorphism $h$ of the topological group $C(R, R)$ into the topological group $\mathrm{C}(\mathrm{Z}, \mathrm{R})$ may be extended to a homomorphism $\mathrm{H}$ of the same kind from the topological group $C(R, R)$ into the topological group $C(X, G)$ such that I $\circ H=h$.

\section{REFERENCES}

1. Geoghegan, R., On spaces of homeomorphisms, embeddings, and functions $I$, Topology, Vol. 11 (1972), 159-177.

2. Gillman, L. and Jerrison, M., Rings of continuous functions, Van Nostrand, N. Y. (1960).

3. Larsen, R., Banach algebra, Dekker, N. Y. (1973).

4. Magill, K. D. Jr., Some Homomorphism theorems for a class of semigroups, Proc. London Math. Soc., Vol. 15 (1965), 517-526.

5. Nickolas, P., Free topological groups and function spaces, Preprint. 
6. Yang, J. S., Transformation groups of automorphisms of $C(X, G)$, Proc. Amer. Math. Soc. Vol. 39 (1973), 619-624; erratum, ibid Vol. 48 (1975), 518.

7. Yang, J. S., On isomorphic groups and homeomorphic spaces, Proc. Amer. Math. Soc. Vol. 43 (1974), 431-438; erratum, ibid, Vol. 48 (1975), 518. 


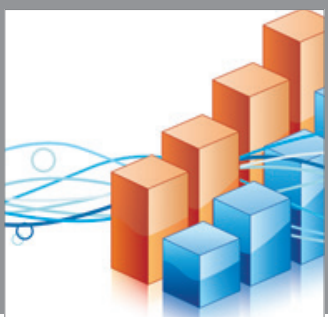

Advances in

Operations Research

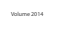

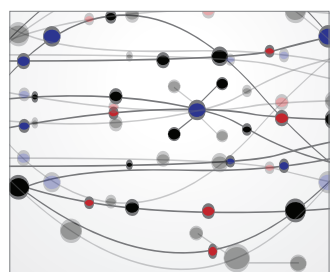

\section{The Scientific} World Journal
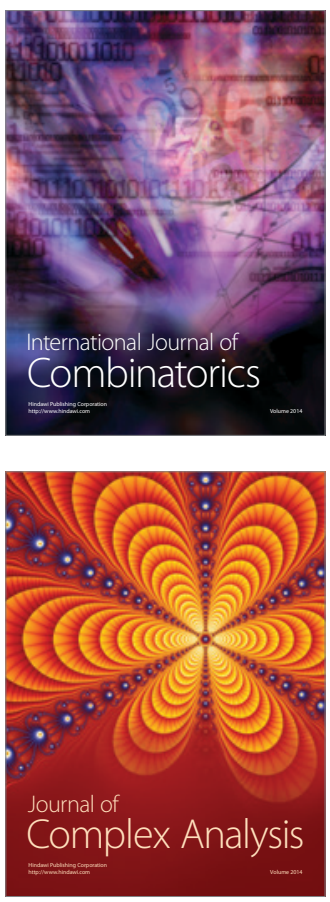

International Journal of

Mathematics and

Mathematical

Sciences
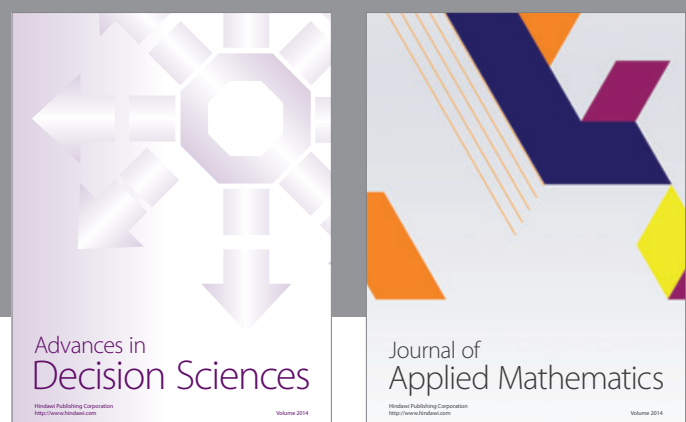

Journal of

Applied Mathematics
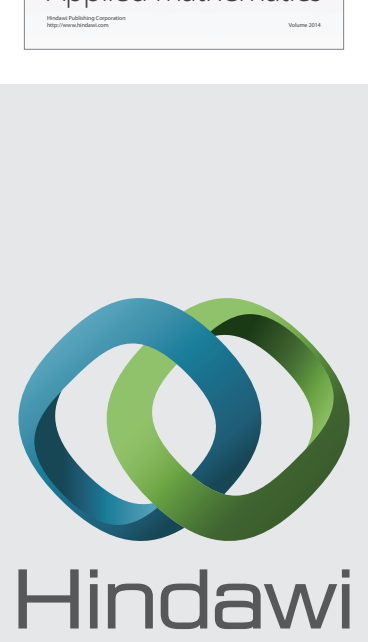

Submit your manuscripts at http://www.hindawi.com
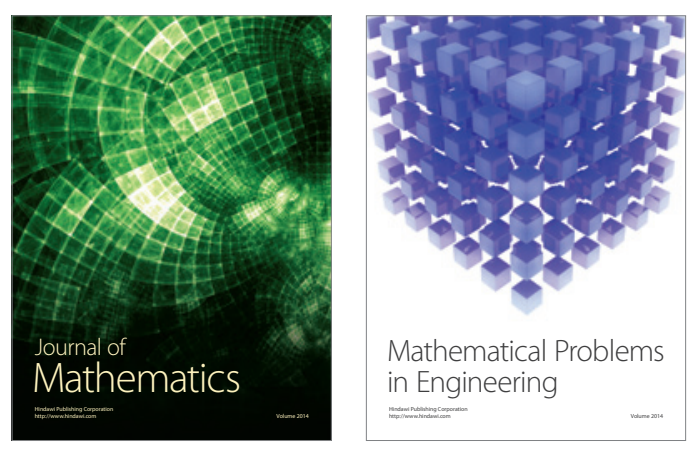

Mathematical Problems in Engineering
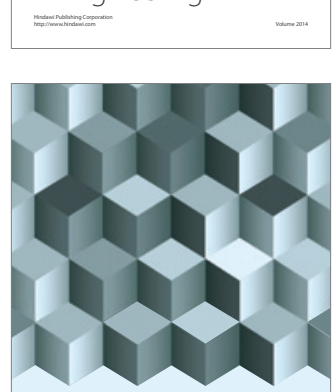

Journal of

Function Spaces
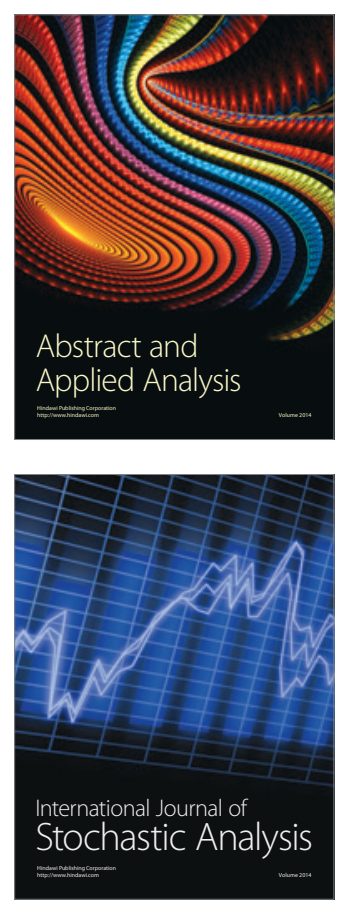

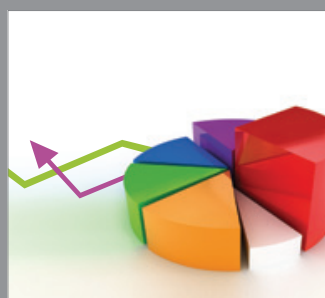

ournal of

Probability and Statistics

Promensencen
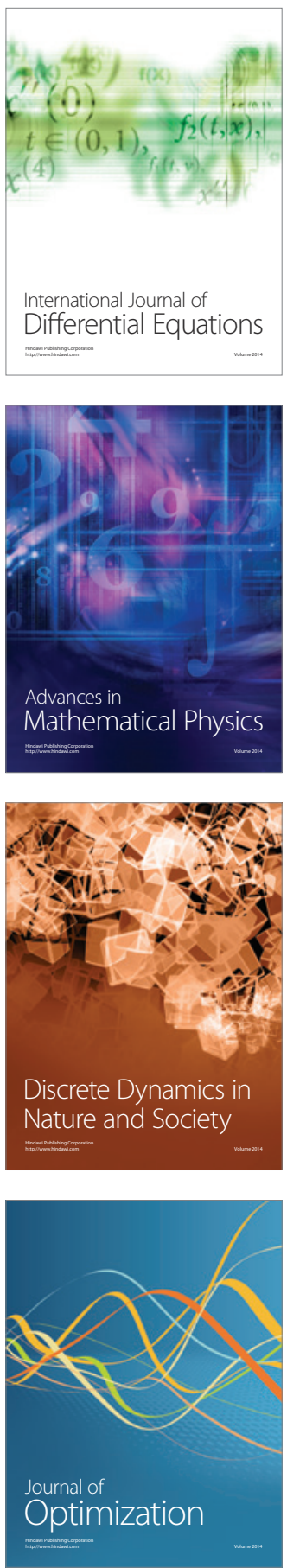